\title{
Kernos
}

Revue internationale et pluridisciplinaire de religion grecque antique

20 | 2007

Varia

\section{CALAME Claude, Pratiques poétiques de la mémoire. Représentations de l'espace-temps en Grèce ancienne}

\section{Vinciane Pirenne-Delforge}

\section{(2) OpenEdition}

\section{Journals}

Édition électronique

URL : https://journals.openedition.org/kernos/337

DOI : 10.4000/kernos.337

ISSN : 2034-7871

\section{Éditeur}

Centre international d'étude de la religion grecque antique

\section{Édition imprimée}

Date de publication : 1 janvier 2007

Pagination : 407-409

ISSN : 0776-3824

\section{Référence électronique}

Vinciane Pirenne-Delforge, " CALAmE Claude, Pratiques poétiques de la mémoire. Représentations de l'espace-temps en Grèce ancienne », Kernos [En ligne], 20 | 2007, mis en ligne le 18 juin 2011, consulté le 08 septembre 2022. URL : http://journals.openedition.org/kernos/337 ; DOI : https://doi.org/ $10.4000 /$ kernos.337 
Le dernier mot est pour affirmer modestement que ce livre n'est qu'« un prologue, l'ouverture d'un chemin à suivre, mais déjà tracé depuis des siècles ». On ne pouvait mieux dire. Si beaucoup de perspectives sont ouvertes ici, il arrive de fait qu'elles soient parfois rapidement refermées. La matière est d'ailleurs immense, comme l'A. l'a bien pressenti. Au passage, elle évoque, par exemple, le poète-philosophe Parménide qui est aussi de cette époque et l'usage symbolique qu'il fait de l'image du chemin; il eût fallu à tout le moins citer l'ouvrage de L. Couloubaritsis (Mythe et philosophie chez. Parménide, Bruxelles, 1990²) dont l'interprétation fait une place décisive à ce symbole. Les éléments de réflexion que Ph.G. propose sont de nature à enrichir grandement le traitement du thème pèlerin en Grèce. À l'inverse d'ailleurs, elle aurait pu tirer elle-même profit d'études déjà parues sur le sujet; on pense notamment aux théories que les Athéniens envoyaient à Délos et à Delphes, sur les traces respectivement d'un héros ou d'un dieu ( $f$., par ex., G. Siebert, dans Les Pèlerinages dans l'Antiquité classique et biblique, Paris, Geuthner, 1973, p. 33-53, et ma contribution dans Histoire des pèlerinages non chrétiens, Paris, Hachette, 1987, p. 94-135). Pour d'autres matières, la bibliographie est en revanche étoffée (environ 230 titres au total). Un index eût été bien utile. Mais saluons avant tout l'originalité de l'entreprise et son audace pluridisciplinaire.

André Motte

(Université de Liège)

CALAME Claude, Pratiques poétiques de la mémoire. Représentations de l'espace-temps en Grèce ancienne, Paris, La Découverte, 2006. 1 vol. 13,5 × $22 \mathrm{~cm}, 322$ p. (Textes à l'appui. Série Histoire classique). ISBN : 2-7071-4798-1.

Comme l'indique le sous-titre, cet ouvrage est une réflexion sur les représentations grecques de l'espace et du temps. Toutefois, contrairement aux perspectives philosophiques qui ont souvent marqué de telles investigations, c'est une analyse des pratiques discursives de différents matériaux poétiques qui est ici privilégiée. Il s'agit, par l'analyse de quatre " configurations textuelles » particulières, de comprendre "la représentation discursive du temps dans sa logique propre et en relation avec l'espace de son développement» (p. 83). L'approche méthodologique se déploie dans un long chapitre introductif qui pose également le cadre des manières contemporaines d'aborder les concepts d'espace et de temps. C.C. offre tout d'abord une critique, brève mais incisive, de l'approche heideggerienne du temps. Ensuite, dans le cadre de l'analyse, exigeante, de son appropriation et de son dépassement par P. Ricœur, c'est la question de l'histoire qui est posée. L'histoire est à la fois savoir partagé sur le passé, pratique discursive, et perception et représentation du passé, ces trois modalités de l'histoire se trouvant en perpétuelle interaction. En effet, l'histoire comme savoir se nourrit de la représentation du passé et le savoir partagé ne reste pas imperméable au remodelage discursif de l'histoire comme pratique. À cet égard, le domaine grec est tout particulièrement intéressant puisque nous situons là les premiers moments d'une histoirepratique discursive dont l'A. souligne l'intimité avec la conception classique de la fabrique poétique. Cette intimité n'est pas contredite, comme on l'affirme souvent, par le célèbre passage d'Aristote comparant poésie et histoire (Poétique, 1451b 27-32, commenté p. 62-63). Dès lors, l'historiographie devient une « historio-poiésis » et l'espace est aussi important que le temps dans l'activité « configurante» des premiers logographes. Mais la dimension énonciative de la mise en discours historiographique n'est pas déterminante seulement pour appréhender le travail de «l'historiopoiète » grec : nos pratiques contemporaines n'y échappent pas et la conscience de cette complexité permet de contextualiser - et de relativiser aussi nos productions d'aujourd'hui en évitant de les figer en savoirs immuables... C'est donc la construction symbolique et discursive du temps et de l'espace qui est étudiée dans 
les quatre dossiers envisagés qui reprennent et/ou prolongent des études antérieures de l'A. : le «mythe des races » d'Hésiode, le 17e dithyrambe de Bacchylide et l'épreuve probatoire de Thésée, la fondation légendaire de Cyrène utilisée à des fins identitaires et, enfin, les lamelles d'or « orphico-dionysiaques ». C'est dire que sont convoquées au cœur de ce livre les deux notions centrales qu'affronte en permanence l'historien de la religion grecque : le mythe et le rite. De plus, en ouvrant ces dossiers à une comparaison de type anthropologique, même limitée, C.C. invoque, non pas une quelconque généalogie de sens ou d'improbables « universaux», mais un "triangle critique» qui impose la contextualisation différentielle du terrain étudié et de l'objet de la comparaison, assortie d'un retour critique sur notre propre espace culturel et nos intérêts académiques. Ainsi, C.C. met en évidence, à chaque fois, un point de méthode précis, qui renvoie à l'un ou l'autre paradigme qui a caractérisé les sciences humaines au cours des dernières décennies.

Le chapitre 2 sur la « succession des âges » a déjà été publiée dans le volume 17 de Kernos (2004, p. 67-102) et je ne m'y arrêterai pas. Qu'il me suffise de souligner l'importance de cette étude pour comprendre ce que l'A. ne veut plus appeler «mythe des races» dans l'économie de l'œuvre hésiodique qui le produit, mais aussi l'articulation opérée ensuite par bon nombre d'auteurs grecs ultérieurs, poètes ou «historio-poiètes », entre passé et présent. Quant au paradigme mis en examen, il s'agit bien entendu de l'approche structuraliste qu'avaient proposée du récit hésiodique les études pionnières de J.-P. Vernant.

Le chapitre 3 s'intitule « Fabrication du genre et identité hérö̈que entre légende et culte : la création politique de Thésée par Bacchylide». Le paradigme mis à l'épreuve est celui qu'ont développé les gender studies. Le poème de Bacchylide, qui déploie un récit linéaire dont l'A. livre une analyse sémio-narrative, renvoie in fine au rituel des Delia qui en encadre la récitation et dont la composante féminine est fortement soulignée par les différents auteurs qui l'évoquent. Mais à l'image « initiatique » souvent invoquée pour rendre compte du plongeon de Thésée et que C.C. nuance ici fortement à l'aide d'une incursion comparative en Nouvelle-Guinée, il ajoute à bon droit la dimension cosmologique du récit, en harmonie avec la position même de l'île de Délos. Quant au statut de « jeune mariée » qu'assumerait le jeune homme sortant des ondes couvert des cadeaux d'Amphitrite, c'est un point qui m'a laissée perplexe, sans que le détour par le gender et par la comparaison ne me permette vraiment d'adhérer à cette analyse sophistiquée. En revanche, la conclusion est limpide : C.C voit dans le poème une contribution à la légitimation des visées maritimes impérialistes d'Athènes sur l'Égée puisqu'il place implicitement l'émergence marine de Thésée en regard du surgissement autochtone de Cécrops ou d'Érichtonios.

Le chapitre 4 emmène le lecteur à Cyrène, qui avait occupé une large place de l'ouvrage Mythe et histoire dans l'Antiquité grecque. La création symbolique d'une colonie (1996). Cette fois, il s'agit de revenir sur l'inscription dite du Serment des fondateurs de la cité, dont C.C. livre une analyse discursive. Elle lui permet de conclure que ce document a été formulé au IVe $\mathrm{s}$. pour rencontrer des impératifs politiques particuliers. L'authenticité du texte, pour les Cyrénéens, est donc intimement liée à la consécration du document ainsi « refait » dans le sanctuaire d'Apollon Pythien. Le contrat initial de la fondation est à nouveau conclu entre les citoyens. Le temps et l'espace dont il est ici question sont totalement politiques, au sens le plus large du terme. Et le paradigme mis en cause est celui d'une philosophie idéalisante qui attribue aux Grecs la conception d'un temps cyclique, «décontextualisé », que contredit ce document et bien d'autres sources encore.

Le chapitre 5 se penche sur les lamelles dites «orphico-dionysiaques ». Partant d'une analyse détaillée du texte d'Hipponion, le plus ancien de la série, C.C. soumet ces documents à plusieurs interrogations. Il en questionne à la fois le statut prétendument «orphique » et l'arrière-plan mystique et initiatique que la recherche récente a contribué à mettre en évi- 
dence. De telles lectures pourraient relever d'aspirations néo-mystiques dont C.C. dénonce le caractère syncrétique et bricolé dans une société moderne hypermédiatisée. C'est le quatrième paradigme mis en cause. La réponse à la question du statut orphique de ces textes est négative, dans la mesure où, pour C.C., les graffiti d'Olbia ne sont pas pertinents dans ce contexte, pas plus que l'iconographie des vases apuliens ou le papyrus de Gurôb habituellement appelés à la barre pour soutenir cette lecture orphique. Les défunts accompagnés par les lamelles ont dû se soumettre de leur vivant à des cérémonies initiatiques du type de celle d'Éleusis (ce qui impliquerait des cultes publics), mais avec Dionysos comme dieu principal. Il n'est question ni de transmigration des âmes ni de réincarnation «orphique », mais d'un parcours rituel ponctué d'indications d'ordre pratique fondant un avenir glorieux à accomplir dans l'éternité, dont le modèle est héroïque.

Bon nombre des positions adoptées par C.C. vont à contre-courant d'une certaine doxa, ce dont il est parfaitement conscient. Reconnaissons toutefois que l'armature théorique de cet ouvrage et l'exigence critique dont elle témoigne en décourageront sans doute plus d'un. La doxa a encore de beaux jours devant elle, mais tout historien de la religion grecque qui aborde la question du «mythe», des «rites de passage » ou de l'« orphisme» doit tenir compte des propositions de C.C. qui a, en outre, le grand mérite de tisser des liens entre antiquité et $\mathrm{XXI}^{\mathrm{e}}$ siècle, comme le montre encore la conclusion du livre.

Vinciane Pirenne-Delforge (FNRS - Université de Liège)

PACHE Corinne Ondine, Baby and Child Heroes in Ancient Greece, Urbana / Chicago, University of Illinois Press, 2004. 1 vol., 15,5 × 23,5 cm, X+234 p. (Traditions). ISBN: 0-252-02929-1.

Le monde des héros comporte une catégorie à part, celle des enfants prépubères disparus prématurément de mort violente, volontaire ou accidentelle, avant d'avoir pu se distinguer par de hauts-faits. Leur mort est pourtant expiée par leur héroïsation caractérisée par l'instauration d'un culte souvent lié à la fondation de jeux funéraires. L'oubli dans lequel la recherche les a longtemps tenus est maintenant réparé. Dans cet ouvrage, issu de sa thèse de doctorat à l'Université de Harvard, Corinne Ondine Pache dresse un inventaire détaillé des sources littéraires, iconographiques et archéologiques en s'interrogeant sur les motifs du statut particulier de ces enfants dans l'imaginaire collectif. Les documents rassemblés sont répartis sur un arc chronologique très large, d'Homère à l'époque romaine.

On pourrait inscrire ce travail dans la mouvance d'une double tendance de la recherche actuelle. D'un côté, il participe à l'approfondissement de nos connaissances sur la place de l'enfant dans la société antique, possible uniquement grâce à une approche interdisciplinaire. De l'autre, il explore un champ relativement nouveau, celui de l'histoire des émotions et des sentiments privés qui ne sont pas directement accessibles dans les sources traditionnelles, mais que l'on peut tenter de lire en creux au travers des mythes et des pratiques rituelles.

L'ouvrage se compose de six chapitres consacrés aux enfants de Médée et d'Héraclès, à Linos et Démophon, Pélops, Opheltès-Archémoros ainsi qu’à Mélicerte-Palaemon, comparés aux destins contrastés d'autres enfants mythiques qui ne deviennent pas des héros, tels le petit Glaucos, le fils de Minos qui se noie dans une jarre de miel puis ressuscite, ou les enfants de Niobé, ensevelis à Thèbes, mais sans faire l'objet d'un culte.

L'introduction retrace l'historique des recherches antérieures sur les cultes hérö̈ques en relevant la nécessité de croiser des sources de nature variée pour saisir la nature et le rôle des enfants où l'A. englobe tous ceux qui appartiennent à la classe d'âge désignée par les termes paidion ou pais. 